\title{
Aromatización de metano sobre Mo/HZSM-5/bentonita: Reactor de lecho fluidizado convencional frente a reactor de lecho fluidizado de dos zonas
}

\author{
J. Lasobras, J. Soler, J. Herguido, M. Menéndez \\ Grupo de Catálisis, Separaciones Moleculares e Ingeniería de Reactores (CREG) \\ Instituto de Investigación en Ingeniería de Aragón (I3A). \\ Universidad de Zaragoza, Mariano Esquillor s/n, 50018, Zaragoza, Spain. \\ Tel. +34-976762707, Fax +34-976762043, e-mail: jlasobra@unizar.es
}

\section{Resumen}

Se ha realizado la transformación de metano a productos aromáticos utilizando un catalizador de Mo/HZSM-5/bentonita. Este proceso tiene el inconveniente de la desactivación del catalizador por la deposición de coque. En este trabajo se propone la configuración de reactor de lecho fluidizado de dos zonas (RLFDZ) para eliminar este inconveniente y permitir la operación en continuo.

\section{Introducción}

La aromatización de metano se presenta como una alternativa al aprovechamiento del gas natural mediante la transformación del $\mathrm{CH}_{4}$ en un producto de mayor valor añadido y más fácil manejo como es el benceno, líquido a temperatura ambiente [1]. Se encuadraría por tanto dentro de las técnicas GTL (gas to liquid). La reacción consta de varias etapas, cada una dependiente de un componente del catalizador. Además, se obtienen productos secundarios como $\mathrm{H}_{2} \quad \mathrm{o}$ coque, este último responsable directo de la desactivación del catalizador [2]. Para evitar esta desactivación se introduce el concepto de reactor de dos zonas que permite regenerar in situ el catalizador por la adición de un oxidante [3].

La reacción ha sido ampliamente estudiada, tanto desde el punto de vista del catalizador, como variaciones en el sistema experimental [4]. De entre los catalizadores, el compuesto por ZSM-5, molibdeno y bentonita es el óptimo para el uso en sistemas de reactores fluidizados.

\section{Experimental}

Los ensayos se realizaron con catalizador preparado con HZSM-5 como función ácida, molibdeno como función metálica y bentonita como aglomerante y encargado de aportar resistencia mecánica.

Se realizaron experimentos en Reactor de Lecho Fluidizado Convencional (RLFC) y Reactor de lecho fluidizado de dos Zonas (RLFDZ), con una velocidad espacial similar. Existen dos diferencias entre las dos configuraciones: la forma de alimentar los reactivos al sistema y la incorporación de un agente oxidante en el caso del RLFDZ. En la Figura 1 se muestran las dos configuraciones y se observa como en el RLFDZ se distinguen dos zonas diferentes. Las dos zonas se diferencian por la atmósfera en la que se encuentra el catalizador: reductora en la zona superior y oxidante en la inferior. La corriente resultante de la reacción se analizó por cromatografía de gases.

La reacción se llevó a cabo con una corriente de gas de $\mathrm{CH}_{4} / \mathrm{N}_{2}$ para el RLF y $\mathrm{CH}_{4} / \mathrm{N}_{2}+\mathrm{O}_{2}$ en RLFDZ a $700{ }^{\circ} \mathrm{C}$ en reactores de cuarzo.

\section{Resultados y discusión}

Los resultados de ambos experimentos a lo largo del tiempo se muestran en las Figuras 2-4. La conversión es superior para el caso del RLFC, sin embargo, se observa una marcada desactivación debida a la formación de coque que se deposita sobre el catalizador. Para el caso del RLFDZ no se ve esta desactivación, sino que se observa una conversión constante con el tiempo. Este hecho no se debe a que no exista deposición de coque, sino que al añadir un agente oxidante, este coque es eliminado y el catalizador permanece activo.

La selectividad a hidrocarburos aromáticos (BTX) en relación a los hidrocarburos producidos, es igual para ambos reactores. Sin embargo, el producto principal (benceno) presenta una selectividad algo inferior para el RLFDZ. Esta diferencia se debe a la aparición de un nuevo producto, el CO proveniente de la reacción del coque con el $\mathrm{O}_{2}$, lo que regenera del catalizador. Otro modo de ver la desactivación del catalizador es en los productos intermedios de la reacción: los $\mathrm{C}_{2}$. El hecho de que aumente la selectividad a $\mathrm{C}_{2}$ con el tiempo es el resultado del bloqueo de los sitios de ciclación por los depósitos carbonosos situados sobre ellos. 


\section{Conclusiones}

La reacción de aromatización de metano puede llevarse a cabo de modo continuo en un RLFDZ. La menor selectividad al producto principal, benceno, se ve compensada por la existencia de una zona de regeneración en el reactor, que facilita el mantenimiento de un catalizador activo, ya que se elimina en la zona oxidante el coque generado en la zona de reacción. Se evita la parada del sistema y el cambio o regeneración del catalizador lo que implicaría un trabajo en ciclos.

\section{REFERENCIAS}

[1]. WANG, L.S., TAO, L.X., XIE, M.S., XU, G.F., HUANG, J.S. and XU, Y.D. Dehydrogenation and

a)

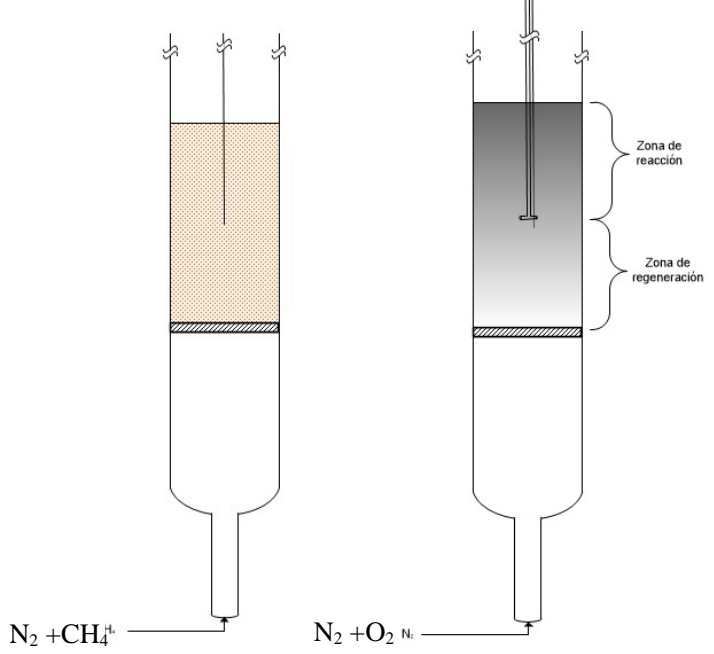

Figura 1: Configuración de reactores a) RLFC y b) RLFDZ

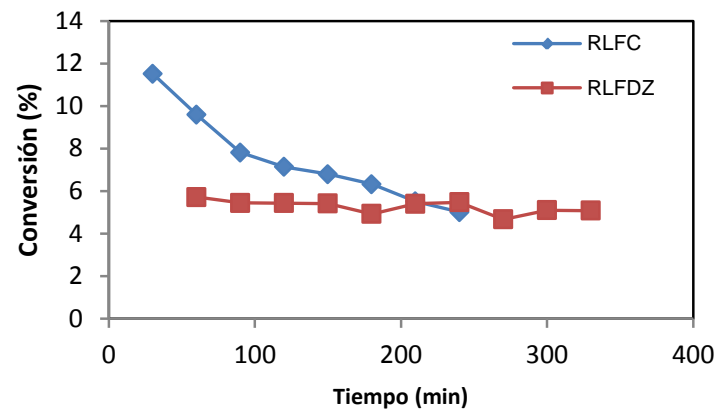

Figura 2: Comparativa entre RLF y RLFDZ de la evolucion temporal de la conversión en la aromatización de metano sobre Mo/HZSM-5/bentonita a $700{ }^{\circ} \mathrm{C}$
Aromatization of Methane Under Nonoxidizing Conditions. Catalysis Letters. 1993, 21(1-2), 35-41.

[2]. WONG, K. S., THYBAUT, J. W., TANGSTAD, E., STÖCKER, M. W. and MARIN, G. B. Methane aromatisation based upon elementary steps: Kinetic and catalyst descriptors. Microporous and Mesoporous Materials. 2012, 164, 302-312.

[3]. GIMENO, M., SOLER, J., HERGUIDO, J. and MENENDEZ, M. Counteracting Catalyst Deactivation in Methane Aromatization with a Two Zone Fluidized Bed Reactor. Industrial \& Engineering Chemistry Research. 2010, 49(3), 9961000 .

[4]. MAJHI, S., MOHANTY, P., WANG, H. and PANT, K.K. Direct conversion of natural gas to higher hydrocarbons: A review. Journal Of Energy Chemistry. 2013, 22(4), 543-554.

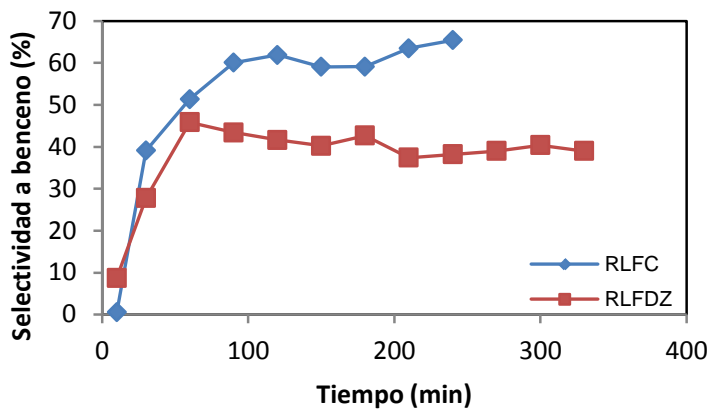

Figura 3: Comparativa entre RLF y RLFDZ de la evolucion temporal de la selectividad a benceno en la aromatización de metano sobre Mo/HZSM-5/bentonita a $700{ }^{\circ} \mathrm{C}$

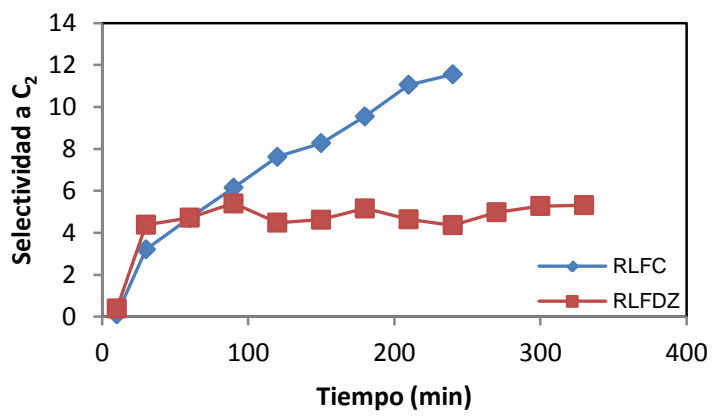

Figura 4: Comparativa entre RLF y RLFDZ de la evolucion temporal de los $\mathrm{C}_{2}$ en la aromatización de metano sobre Mo/HZSM-5/bentonita a $700{ }^{\circ} \mathrm{C}$ 